\title{
Editorial: A dearth of apps for psychology: the mind, the phone, and the battery
}

\author{
Samuel Ken-En Gan ${ }^{1,2,3^{*}}$ and Benjamin Yi-Liang Goh ${ }^{1,3}$
}

\begin{abstract}
"Oedipus complex", "penis-envy", "oral fixation", and "interpreting dreams". From the peering into the unconscious mind in the days of Sigmund Freud (Freud, 1940) to the deep questions of evil (Haney, Banks, Jaffe \& Zimbardo., 1972; Milgram 1978), the field of Psychology has been enigmatic since its birth. Despite having clear useful applications (e.g. lie-detection, people management, etc.), the field is still regarded by many to be an 'art' rather than 'science'. While psychology often relied on qualitative observation (Denzin \& Lincoln, 2009), the field also utilized quantitative experimental analysis (see example: Brown \& 21 Ryan, 2003), which had benefited from the use of computer programs e.g. E-Prime (Schneider et al., 2002), SAS Institute (2004), etc. The uses of these software are tantamount to high accuracy of data collected in certain sub-disciplines such as cognitive or neuropsychology where cognitive speed and response time are measured (see example: Truedsson et al. 2015.) More commonly, the use of surveys are highly prevalent and can be used to gather quantitative selfreported data (see examples: Gan, Lim, \& Haw 2015; Yew et al. 2015) due to their ease of administration.
\end{abstract}

Although the use of "pen and paper" approach previously dominated data collection, modern day analysis and collection of data are increasingly performed by software that are not only more convenient, but give more accurate measurements. While these software expectedly help to calculate and crunch numbers for statistical analysis in quantitative research, they can also be used in qualitative research. Such qualitative targetted software include speech-to-text software for transcription of qualitative interviews, and video analysis software for body languages (Koch \& Zumbach 2002).

Although useful, these computer programs and equipment also spatially bound researchers and participants

\footnotetext{
*Correspondence: samuelg@bii.a-star.edu.sg

${ }^{1}$ Bioinformatics Institute, Agency for Science, Technology, and Research

(A*STAR), Singapore 138671, Singapore

${ }^{2}$ p53 Laboratory, Agency for Science, Technology, and Research (A*STAR),

Singapore 138648, Singapore

Full list of author information is available at the end of the article
}

by virtue of their immobility. The convenience is also further limited by the lack of suitable places for long interviews and psychological batteries (long psychology surveys with hundreds of items).

Fortunately, despite all these disadvantages, one modern invention holds the potential to revolutionize these practices - The Smartphone.

Two decades ago, the first smartphone by IBM captured everyone's attention with its ability to incorporate the multiple functions into one small brick-like device. However, due to the price tag, only about 50,000 units were sold worldwide (Sager, 2012). However, technological advancements have since reduced cost, and its affordability now makes it prevalent throughout developed countries worldwide. These smartphones now act as mini-computers that can function on a variety of operating systems (OS) such as Android, iOS, Windows, Blackberry OS, etc. Like the desktop, every OS has their own unique market for applications and these have percolated to many areas of the modern life from travel to tracking family members and friends. Despite this, there is a dearth of apps to meet the needs of experimental psychology. This thus shows a gap for the commercial and academic development of psychological smartphone apps.

\section{Nature and limitations of psychological research}

Experimental psychology typically involves studying the effect of certain stimuli. These can include environmental stimuli in the natural setting (see example: Kööts et al. 2015) to experimentally induced parameters (see example: Williams \& Bargh 2008). As it is not always possible to know what exactly happens in the mind nor measure things physiologically (one cannot easily measure happiness or sadness quantitatively), it is common to assess some responses through self-reports via surveys or psychological batteries. Nonetheless, there are glaring limitations that impede such data collection.

A typical psychology battery can consist of hundreds of items (questions) and can take hours to fully respond. In the fast-paced society of today, long data collection

\section{Springer}

(c) 2016 Gan and Goh. Open Access This article is distributed under the terms of the Creative Commons Attribution 4.0 International License (http://creativecommons.org/licenses/by/4.0/), which permits unrestricted use, distribution, and reproduction in any medium, provided you give appropriate credit to the original author(s) and the source, provide a link to the Creative Commons license, and indicate if changes were made. 
times are highly unpopular with participants, resulting in high dropout rates and low sample numbers. A possible solution to this is to increase accessibility and convenience of survey participation using smartphone applications, and in a way that participants can complete them at their convenience (see example app: PsychVey, described in Nguyen et al. 2015).

The other difficulty lies in measuring individual data. Current psychological research relies too heavily on selfreports which are highly subjective and biased (Dunning et al. 2004). These self-reports are clouded by denial, moods, and cognitive dissonance (see example: Spector, 1994). While there are equipment to directly measure some physiological parameters (such as cortisol, see Van Cauter et al. 1996), these resources are not widely accessible to the general public due to the requirement of skilled use. skilled use. Again, with the smartphone to the rescue, it may be possible to leverage on sensors (either in-built into smartphones or peripherally attached) to simplify the skills and make common the ability to collect such direct measurments. This allows individual qualitative data to be collected quantitatively. One way is to use the microphone to measure heart rate (iStethoscope in Apple App Store) for anxiety studies. Such measurements get far more accurate data than self-reports of anxiety, especially if the participants have tendencies to underplay or overplay their difficulties (such as in the case of hypochondriacs).

The final limitation of psychological studies, and also the most important problem, is the lack of diversity in the sample size. It is often only possible to investigate and study a local sample because of the geographical constraints. As a result, studies are often culture-specific and lack ecological validity, making it difficult for the study to be generalized to real-life settings. By using smartphone apps to gather data (this can take the form of surveys, physiological measurements or cognitive responses), data can be collected from across the globe easily and cost effectively, making psychological findings more relevant to a wider general audience. With larger sample sizes that are more diverse, psychological studies may be more reproducible, a problem recently highlighted (Open Science Collaboration, 2015).

\section{Current psychology apps}

A search on Google Play store using keywords 'psychology', 'psychoanalysis,' 'psychology experiments' returned mostly apps that are books. Some of these apps are mock examinations, e.g. 500 AP Psychology Questions by gWhiz (2013) and AP Exam Prep Psychology by K12 Inc (2014). Many of the others are unvalidated psychological measures that claim to predict personality traits or relationship status. Even meditation guides are found amongst these apps. Irregardless, there is a general dearth of apps that directly aid psychological research.

Fortunately, when searching for specific psychological tests such as "Stroop", "Tower of Hanoi", and "Memory", there are thousands of apps. While many of these are great apps and some allow comparison of scores, they typically function as games and puzzles, and have yet to be prominently exploited for research purposes. It would certainly be a data mine for psychology researchers should the data of the scores and demographic data (with permission for use) be collected for meta-analysis to establish international standards and norms.

\section{The future: New applications}

Experimental Psychology has significant overlaps with clinical and biomedical research. As apps for the mentioned fields are more abundant than psychology at present, it is certainly possible to use apps in these areas for psychological purposes. Already mentioned above, anxiety could be measured using apps for clinical measurements (e.g. heart-rate).

With increasingly powerful cameras, smartphone can now double up as recording devices for investigations into micro expressions and body language in social psychology research. Similarly, the in-built microphone can be used for voice stress pattern or be used to measure certain physiological effects. Already used in some of the Stroop experiment apps, the high sensitivity touch screen of the smartphone measures response time, which can easily be expanded to include experiments of miscue, priming, and judgements.

Through games and puzzles for cognitive psychology, response times and mental alertness are already measured. These apps can collect data from the longitudinal monitoring of memory and other cognitive capabilities. These are likely to also have clinical relevance as patients with Parkinson's disease perform poorer in games that require precise motor controls (dos Santos Mendes et al. 2012) and the collected data can contribute to pre-diagnosis without subjecting the patients to the series of long and tedious assessments. Furthermore, games which focuses on balance training could improve the mobility and functional abilities of Parkinson's patients (Esculier et al. 2012), making these apps part of physiotherapy.

The potential of phone apps in psychology is further expanded by the peripheral add-ons that can be linked to the smartphone wirelessly (WIFI, Bluetooth, NFC, etc.). This creates more opportunities for direct physiological or even neuropsychological parameters to be measured. It may be possible for electroencephalography (EEG) probes to send readings to mobile devices for the monitoring of brain activity. Other sensors that detect skin conductance, blood pressure and heart rates used in 
sports can also be tapped on for biopsychological research.

In conclusion, the research possibilities that mobile applications provide for the field of psychology are wide and exciting. By embracing these technological advancements that are already easily available to us, we can bring much convenience into the lives of the psychologist, patients, and the general public. But we need the collective effort of psychologists, app developers and engineers to realise the potential of phone apps in psychology to grow not only the use of scientific phone apps and mobile devices, but also psychological research.

\section{Author contributions}

BYL drafted the manuscript. SKEG co-wrote the manuscript. All authors have read and approve of the final manuscript.

\section{Acknowledgements}

We would like to thank Cornelius Koshy for helping with the proof-reading.

\section{Author details}

${ }^{1}$ Bioinformatics Institute, Agency for Science, Technology, and Research (A*STAR), Singapore 138671, Singapore. ${ }^{2}$ p53 Laboratory, Agency for Science, Technology, and Research (A*STAR), Singapore 138648, Singapore. ${ }^{3}$ James Cook University, Singapore 387380, Singapore.

Received: 6 January 2016 Accepted: 6 January 2016

Published online: 02 April 2016

\section{References}

Brown KW, Ryan RM. The benefits of being present: mindfulness and its role in psychological well-being. J Pers Soc Psychol. 2003;84(4):822.

Denzin NK, Lincoln YS. Qualitative research. Yogyakarta: PustakaPelajar; 2009.

dos Santos Mendes FA, Pompeu JE, Lobo AM, da Silva KG, de Paula Oliveira T,

Zomignani AP, et al. Motor learning, retention and transfer after virtualreality-based training in Parkinson's disease-effect of motor and cognitive demands of games: a longitudinal, controlled clinical study. Physiotherapy. 2012;98(3):217-23.

Dunning D, Heath C, Suls JM. Flawed self-assessment implications for health, education, and the workplace. Psychol Sci in public interest. 2004:5(3):69-106.

Esculier JF, Vaudrin J, Beriault P, Gagnon K, Tremblay LE. Home-based balance training programme using Wii Fit with balance board for Parkinson's disease: a pilot study. J Rehabil Med. 2012;44(2):144-50.

Freud, S. (1940). An outline of psychoanalysis.

Gan, S.K.E., Lim, M.J., Haw, Y.X. (2015) The Relaxation Effects of Stimulative and Sedative Music on Mathematics Anxiety: A Perception to Physiology Model. Psychology of Music. doi:10.1177/0305735615590430

gWhiz. (2013). 500 AP Psychology Questions (1.3) [Mobile application software] Retrieved from https://play.google.com/store/apps/details?id=com. gwhizmobile.psych500\&hl=en.

Haney, C., Banks, C., Jaffe, D. and Zimbardo, P., 1972. Stanford Prison Experiment.

K12 Inc. (2014). Ap Exam Prep Psychology (Version 1.2.0) [Mobile application software]. Retrieved from https://play.google.com/store/apps/details?id=air. com.k12.ExamPrepPsych\&hl=en.

Koch, S. C., \& Zumbach, J. (2002, May). The use of video analysis software in behavior observation research: Interaction patterns in task-oriented small groups. In Forum Qualitative Sozialforschung/Forum: Qualitative Social Research (Vol. 3, No. 2).

Kööts, L., Realo, A., \& Allik, J. (2015). The influence of the weather on affective experience. Journal of individual differences.

Milgram, S. (1978). Obedience to authority

Nguyen PV, Lim JPH, Budianto IH, Gan SKE. PsychVeyApp: research survey app. Scientific phone apps and mobile devices, 1(1), 1-4

Open Science Collaboration, 2015. Estimating the reproducibility of psychological science. Science, 349(6251), p.aac4716.

Sager, I. (2012). Before IPhone and Android came Simon, the first smartphone. Bloomberg Businessweek, 29.
SAS Institute. (2004). SAS/GRAPH 9.1 Reference. SAS Institute.

Schneider, W., Eschman, A., \& Zuccolotto, A. (2002). E-Prime: User's guide. Psychology Software Incorporated.

Spector PE. Using self-report questionnaires in OB research: A comment on the use of a controversial method. J Organ Behav. 1994;15(5):385-92.

Truedsson, E., Bohlin, G., \& Wåhlstedt, C. (2015). The Specificity and Independent Contribution of Inhibition, Working Memory, and Reaction Time Variability in Relation to Symptoms of ADHD and ASD. Journal of attention disorders, 1087054715587093

Van Cauter E, Leproult R, Kupfer DJ. Effects of gender and age on the levels and circadian rhythmicity of plasma cortisol. J Clin Endocrinol Metab. 1996;81(7):2468-73.

Williams LE, Bargh JA. Experiencing physical warmth promotes interpersonal warmth. Science. 2008;322(5901):606-7.

Yew, S. H., Lim, K. M. J., Haw, Y. X., \& Gan, S. K. E. (2015). The Association between Perceived Stress, Life Satisfaction, Optimism, and Physical Health in the Singapore Asian context. Asian Journal of Humanities and Social Sciences (AJHSS), 3(1)

\section{Submit your manuscript to a SpringerOpen ${ }^{\circ}$ journal and benefit from:}

- Convenient online submission

- Rigorous peer review

- Immediate publication on acceptance

- Open access: articles freely available online

- High visibility within the field

- Retaining the copyright to your article

Submit your next manuscript at $\boldsymbol{s p r i n g e r o p e n . c o m ~}$ 